\title{
New in the diagnosis of heart failure and management of associated conditions in 2021 ESC guidelines
}

Heart failure (HF) is a major health problem affecting millions of people worldwide. The $r$ report about heart disease and stroke statistics claimed that almost 6.0 million Americans $\geq 20$ years of age had HF based on the results of 2015-2018 The National Health and Nutrition Examination Survey (NHANES) (1). This also points to an increase from previous number which was $\approx 5.7$ million according to NHANES 2009 to 20121 . HF prevalence is expected increase by \%46 from 2012 to 2030 . The percentage of population having $\mathrm{HF}$ in 2012 was $2.4 \%$ but it is assumed to increase to $3 \%$ in 2030.

Guideline from European Society of Cardiology (ESC) for the diagnosis and treatment of acute and chronic HF was released recently (2). The prevention side of $\mathrm{HF}$ management was covered as providing a general advice. Any health care provider can use this guideline for helping people with HF with best evidence available. We tried to summarize what is new in diagnosis and management of special conditions in the guideline.

The new 2021 ESC Guidelines for the diagnosis and treatment of acute and chronic $\mathrm{HF}$ as the previous edition from 2016 use the main terminology to describe HF based on measurement of the left ventricular ejection fraction (LVEF). Differentiation of patients with HF based on LVEF is still important due to different underlying demographics, etiologies, comorbidities and response to therapies. HF comprises a wide range of patients, from those with preserved LVEF, typically considered as $\geq 50 \%$ (HFpEF) to those with reduced LVEF considered as $\leq 40 \%$ (HFrEF). In contrast to the previous recommendations from 2016 patients with LVEF in the range of $40-49 \%$ represent a group now named 'heart failure with mildly reduced ejection fraction' (HFmrEF), not 'heart failure with mid-range ejection fraction'. This renaming was supported by the fact that retrospective analyses from recently completed randomized controlled trials in HFrEF that have included patients with ejection fractions in the $40-50 \%$ range suggest that they may also benefit from similar therapies to those with LVEF less than $40 \%$. So abbreviation remains the same (HFmrEF) but now we say 'mildly reduced', not 'midrange'.
Therefore, HF patients are classified to three main groups based on their LVEF.

-HF with preserved EF (HFpEF) group with EF value more than $50 \%$

-HF with mildly reduced EF (HFmrEF) group with EF value between $40-49 \%$

-HF with reduced EF (HFrEF) group with EF value lower than- $40 \%$

The ESC long-term registry reported that the majority of heart failure patients $(60 \%)$ have HFrEF followed by HFmrEF (24\%) and HFpEF (16\%) (3).

HF patients generally have two presentations: Chronic $\mathrm{HF}$ (CHF) and acute HF (AHF). The diagnosis of CHF requires the presence of symptoms (breathlessness, fatigue, ankle swelling etc.) and/or signs of HF and objective evidence of cardiac dysfunction. Unfortunately, symptoms and signs could not be used alone to make the diagnosis of $\mathrm{HF}$ due to lack of sufficient accuracy. Following diagnostic tests are recommended in any patients suspected of having heart failure (Class I recommendation).

1. Electrocardiography

2. Natriuretic peptide measurement when available (NT-proBNP $\geq 125 \mathrm{pg} / \mathrm{mL}$ or BNP $\geq 35 \mathrm{pg} / \mathrm{mL}$ increase the likelihood of HF)

3. Transthoracic echocardiography

4. Chest X-ray

5. Routine blood tests for comorbidities, including full blood count, urea and electrolytes, thyroid function, fasting glucose and $\mathrm{HbAlc}$, lipids, iron status.

These tests should be used for confirmation of HF. Further classification of HF (HFpEF, HFmrEF, HFrEF) can be provided by LVEF measurement by echocardiography, cardiac magnetic resonance (cMR), or rarely nuclear tests. CMR, invasive coronary angiography, computed tomography coronary angiography, exercise testing, cardiopulmonary testing, right heart catheterization and endomyocardial biopsy are specific diagnostic tests which should or may be used in appropriate clinical scenarios.

Address for correspondence: Oben Baysan, Guven Hospital Cardiology Clinic, Ankara, Turkey, email: obbaysan@gmail.com; Yuriy Ivaniv, Lviv Medical University, Lviv, Ukraine

Received: 07.09.2021 Accepted: 10.09.2021

Copyright @2021 Heart, Vessels and Transplantation

doi: 10.24969/hvt.2020.273 


\section{Heart failure with reduced EF}

The diagnosis of HFrEF requires the presence of symptoms and/or signs of HF and a reduced LVEF less than $40 \%$. This evaluation is most usually obtained by echocardiography. Details about the quality standards that should be adhered to when determining the presence of reduced LV systolic function by echocardiography can be found in the European Association of Cardiovascular Imaging (EACVI) position paper. It means that for measurement of LVEF the modified biplane Simpson's rule is strongly recommended. LV end diastolic volume (LVEDV) and LV end systolic volume (LVESV) are obtained from apical four- and two-chamber views. This method relies on accurate tracing of endocardial borders. The Teichholz and Quinones methods of calculating LVEF from linear dimensions, as well as a measurement of fractional shortening, are not recommended, as they may result in inaccuracies, particularly in patients with regional LV dysfunction and/or LV remodeling. The quantification of LV volumes and LVEF could be improved by using of three-dimensional echocardiography of adequate quality. Doppler techniques allow the calculation of hemodynamic variables, such as stroke volume index and cardiac output, based on the velocity time integral at the LV outflow tract area. Tissue Doppler parameters (S wave) and deformation imaging techniques (strain and strain rate) have been shown to be reproducible and feasible for clinical use, especially in detecting subtle abnormalities in systolic function in the preclinical stage. As well as the determination of the LVEF, echocardiography can also provide information on other important parameters such as chamber size, eccentric or concentric LVH, regional wall motion abnormalities that may suggest underlying CAD, Takotsubo syndrome, or myocarditis. It is possible to evaluate using echocardiography the RV function, pulmonary hypertension, valvular function, and markers of diastolic function.

If assessment of EF is not possible by echocardiography, then CMR or rarely, nuclear techniques can be employed.

According to the new 2021 Guidelines invasive coronary angiography may be considered in patients with HFrEF with an intermediate to high pre-test probability of CAD and the presence of ischemia in noninvasive stress tests.

Other imaging modalities also could provide clinically important information. Computed tomography coronary angiography should be considered in patients with HF and a low to intermediate pre-test probability of coronary artery disease (CAD) or those with equivocal non-invasive stress tests in order to rule out coronary artery stenosis.

In 2021 guidelines important place is devoted to the cardiac magnetic resonance in patients with HFrEF. First of all this method of heart visualization is recommended for the assessment of myocardial structure and function in those with poor echocardiogram acoustic windows. Also CMR is recommended for the characterization of myocardial tissue in suspected infiltrative disease, Fabry disease, inflammatory disease (myocarditis), LV noncompaction, amyloid, sarcoidosis, and hemochromatosis. Using of late gadolinium enhancement should be considered in dilated cardiomyopathy to distinguish between ischemic and non-ischemic myocardial damage.

Non-invasive stress imaging (CMR, stress echocardiography, SPECT, PET) may be considered for the patients with CAD and HFrEF to assess the myocardial ischemia and its viability who are considered suitable for coronary revascularization. Exercise testing may be considered also to detect reversible myocardial ischemia.

So the new 2021 ESC Guidelines for the diagnosis and treatment of acute and chronic heart failure recommend different visualization methods for the evaluation of patients with HFrEF providing relevant and clinically important information.

\section{Heart failure with mildly reduced $\mathrm{EF}$}

The HFmrEF can be diagnosed with the presence of symptoms and/or signs of $\mathrm{HF}$, a mildly reduced $\mathrm{EF}$ (41-49\%) and high natriuretic peptide levels (BNP $\geq 35$ $\mathrm{pg} / \mathrm{mL}$ or NT-proBNP $\geq 125 \mathrm{pg} / \mathrm{mL}$ ). Other imaging findings that points to the presence of structural heart disease such as increased left atrial (LA) size, left ventricular (LV) hypertrophy, high LV pressures also raise the possibility of HFmrEF. Patients with HFmrEF have characteristics more resemble of HFrEF than HFpEF. They are younger, more commonly men, likely to have CAD (50-60\%).

\section{Heart Failure with preserved EF}

HFpEF patients are older and more often female. Concomitant diseases such as atrial fibrillation (AF), chronic kidney disease, obesity, anemia, hypertension and diabetes mellitus are more common in patients with HFpEF than in those with HFrEF.

This guideline provides a simplified approach to the diagnosis of HFpEF based on the HFA-PEFF diagnostic algorithm (3). 
The presence of HF signs and symptoms, an LVEF $>50 \%$ and objective evidence of cardiac structural and/or functional abnormalities consistent with the presence of LV diastolic dysfunction/raised LV filling pressures are required for the diagnosis.

Cardiac structural, functional and serological abnormalities consistent with the presence of LV diastolic dysfunction/raised LV filling pressures are to be screened. The cut-off values are as following: LV mass index $-\geq 95 \mathrm{~g} / \mathrm{m} 2$ for females and $\geq 115 \mathrm{~g} / \mathrm{m} 2$ for males; relative wall thickness $>0.42$; LA volume index- $>34 \mathrm{~mL} / \mathrm{m} \mathrm{2}$ (in sinus rhythm, SR); E/e' ratio at rest >9; NT-proBNP >125 (SR)or >365 (AF) pg/mL; or $\mathrm{BNP}>35$ (SR) or $>105$ (AF) $\mathrm{pg} / \mathrm{mL}$; a pulmonary artery systolic pressure $>35 \mathrm{mmHg}$; and tricuspid regurgitant jet velocity at rest - $>2.8 \mathrm{~m} / \mathrm{s}$.

\section{The prevention of hospitalization and patient monitoring}

2021 guideline has new recommendations about HF hospitalization prevention and patient monitoring. When available HF patients are enrolled to multidisciplinary HF management program to reduce the risk of HF hospitalization and mortality (Class I recommendation).

Self-management strategies, home-based and/or clinic-based programs, supervised exercise-based cardiac rehabilitation programs and influenza and pneumococcal vaccinations should be used to reduce the risk of HF hospitalization and mortality. (Class Ila recommendation).

Non-invasive home telemonitoring, if available, may be considered for patients with $\mathrm{HF}$ in order to reduce the risk of recurrent $\mathrm{CV}$ and $\mathrm{HF}$ hospitalizations and $\mathrm{CV}$ death (Class $\mathrm{Ilb}$ recommendation). In patients hospitalized for HF attack, it is recommended to exclude any persistence of congestive signs and symptoms before discharge (Class I recommendation). Moreover, evidence based medicines are to be administered before discharge (Class I recommendation).

The patient should be invited to an early follow-up visit 1-2 weeks after discharge to assess the signs of congestion, drug tolerance, start and/or up-titrate the evidence-based therapies (Class I recommendation) .

\section{Advanced Heart Failure}

The term advanced HF points to the persistence of symptoms and signs of HF despite maximal therapy. Mechanical circulatory support or heart transplantation (Class I recommendation) are the main therapeutic options. This guideline states that continuous inotropes and/or vasopressors may be considered in patients with low cardiac output and evidence of organ hypoperfusion as a bridge to mechanical circulatory support or heart transplantation (Class Ilb recommendation).

\section{Acute Heart Failure}

Acute Heart Failure is usually caused by acute decompensation of chronic heart failure or may be the first manifestation of heart failure (new onset). The guideline suggested new clinical presentation types which require urgent and tailored therapy for each of them - acute decompensated heart failure. acute pulmonary edema, isolated right ventricular failure and cardiogenic shock.

\section{Recommendations for management of patents with $\mathrm{HF}$ and comorbidities}

In this 2021 ESC HF guideline, recommendations are given on how to manage co-existing cardiovascular (CV) and non-CV conditions of HF patients. AF, valvular heart disease, diabetes, chronic kidney disease, iron deficiency and other comorbidities frequently co-exist with HF. Integration of specific treatments may have an impact on the clinical course HF. Furthermore, proper treatment of high blood pressure, diabetes and CAD can prevent the development of HF. We will further present what's new in this 2021 ESC HF guideline in terms of comorbidities.

\section{Cardiovascular comorbidities \\ Atrial Fibrillation}

Patients with AF are in special focus in this new HF guideline. The management of patients with concomitant $\mathrm{HF}$ and $\mathrm{AF}$ is recommended as Class I in terms of identification and treatment of possible causes or triggers of AF, management of $\mathrm{HF}$, prevention of embolic events, rate control, rhythm control.

Newly added recommendation for $\mathrm{HF}$ and $\mathrm{AF}$ association in this guideline is;

Long-term treatment with an oral anticoagulant should be considered for stroke prevention in $\mathrm{AF}$ patients with a CHA2DS2-VASc score of 1 in men or 2 in women (Class Ila recommendation).

There are major recommendations in this guideline for $\mathrm{HF}$ and $\mathrm{AF}$ association which differ from 2016. 
These are;

Direct oral anticoagulants (DOACs) become a class I, level of evidence $A$ recommendation for all patients with $\mathrm{AF}, \mathrm{HF}$, and a CHA2DS2-VASc score $\geq 2$ in men or $\geq 3$ in women.

For the prevention of embolic events, DOACs are recommended in preference to vitamin $\mathrm{K}$ antagonists in patients with HF, except in those with moderate or severe mitral stenosis or mechanical prosthetic heart valves (Class I recommendation). Therefore, DOACs are being given preference over vitamin $\mathrm{K}$ antagonists moving from class Ila to class I. Beta-blockers should be considered for short and long-term rate control in patients with $\mathrm{HF}$ and AF (Class Ila recommendation). With this proposal beta-blockers move down from class I to Ila. In patients with worsening and persistence of HF symptoms despite medical therapy because of a clearassociation between paroxysmal or persistent $\mathrm{AF}$ and HF symptoms, catheter ablation should be considered for the prevention of AF (Class Ila recommendation). Catheter ablation moves up from class IIb to class Ila for the cases with clear association.

\section{Chronic coronary syndromes}

The management of patients with HF and chronic coronary syndromes (CCS) is crucial since CAD is the most common cause of $\mathrm{HF}$ in industrialized, middleincome, and increasingly in low-income, countries.

2016 ESC HF guideline was offering a statement that "myocardial revascularization is recommended when angina persists despite treatment with anti-anginal drugs" as a Class I recommendation. However 2021 ESC HF guideline changes this statement as "For the relief of anginal/angina equivalent symptoms, coronary revascularization is recommended to be considered in patients with $\mathrm{HFrEF}, \mathrm{CCS}$, and coronary anatomy suitable for revascularization, despite optimal medical treatment including anti-anginal drugs (Class Ila recommendation).

The new recommendations considering $\mathrm{HF}$ and CCS are as follows;

Coronary artery bypass grafting (CABG) should be considered as the first-choice revascularization strategy, in patients suitable for surgery, especially if they have diabetes and for those with multi-vessel disease (Class Ila recommendation).

In LV assist device candidates needing coronary revascularization, CABG should be avoided, if possible (Class Ila recommendation).

Coronary revascularization may be considered to improve outcomes in patients with HFrEF, CCS, and coronary anatomy suitable for revascularization, after careful evaluation of the individual risk to benefit ratio, including coronary anatomy (i.e. proximal stenosis $>90 \%$ of large vessels, stenosis of left main or proximal LAD), comorbidities, life expectancy, and patient's perspectives (Class IIb recommendation).

Percutaneous coronary intervention may be considered as alternative to CABG, based on Heart Team evaluation, considering coronary anatomy, comorbidities, and surgical risk (Class $\mathrm{llb}$ recommendation).

\section{Valvular Heart Disease}

Four newly added recommendations regarding HF patients with aortic stenosis and/or secondary mitral regurgitation are as follows in $2021 \mathrm{ESC}$ HF guideline. For severe high-gradient aortic stenosis patients, transcatheter aortic valve implantation or surgical aortic valve replacement is recommended to reduce mortality and improve symptoms (Class I recommendation). The choice of intervention is recommended to be done by the Heart Team according to the aspects of the patient and procedure (Class I recommendation).

For the patients with secondary mitral regurgitation, not eligible for surgery and not needing coronary revascularization, who are symptomatic despite optimal medical therapy and who fulfill criteria to achieve a reduction in HF hospitalizations, percutaneous edge-to-edge mitral valve repair should be considered (Class Ila recommendation). However, this statement was a Class $\mathrm{llb}$ recommendation to improve symptoms for the highly symptomatic patients. With this proposal, COAPT and MITRA-FR trials deserved their place in this update as a major change to the guidelines.

\section{Non-cardiovascular comorbidities Diabetes}

ESC HF 2016 guideline recommended empagliflozin as Class Ila recommendation in patients with type 2 diabetes mellitus (T2DM). There is a revised recommendation in this new 2021 ESC HF guideline based on the results of the trials of sodium-glucose co-transporter 2 (SGLT2) inhibitors. Canagliflozin, dapagliflozin, empagliflozin, ertugliflozin or sotagliflozin are recommended to prevent $\mathrm{HF}$ and $\mathrm{CV}$ death and worsening kidney function in patients with type 2 diabetes and $\mathrm{CV}$ disease and/or CV risk factors, or chronic kidney disease (Class I recommendation). 
Dapagliflozin and empagliflozin are also indicated for the treatment of patients with T2DM and HFrEF and sotagliflozin was shown to reduce CV deaths and HF rehospitalizations in patients recently hospitalized for $\mathrm{HF}$ (Class I recommendation). This recommendations urges that all type 2 diabetic HF patients should be treated with an SGLT2 inhibitor.

In the DPP-4 inhibitor trials and met-analyses, their effects on mortality or CV events were found as neutral in patients with diabetes and HF. Therefore, The DPP-4 inhibitor saxagliptin is not recommended in patients with HF (Class III recommendation).

\section{Iron deficiency and anemia}

Iron deficiency and anemia are commonly encountered in HF patients. Independent from anemia, iron deficiency is found in up to $55 \%$ of chronic $\mathrm{HF}$ and in up to $80 \%$ of acute HF patients. For HF patients, iron deficiency is defined as either a serum ferritin concentration $<100 \mathrm{ng} / \mathrm{ml}$ or $100-299$ $\mathrm{ng} / \mathrm{ml}$ with transferrin saturation $<20 \%$ in $\mathrm{HF}$ patients. All patients with $\mathrm{HF}$ are recommended to be periodically screened for anemia and iron deficiency with a full blood count, serum ferritin concentration, and transferrin saturation as Class I recommendation, since it is associated with reduced exercise capacity, $\mathrm{HF}$ re-hospitalizations, high $\mathrm{CV}$ and all-cause mortality. This guideline incorporates AFFIRM-AHF trial, recommending intravenous iron supplementation with ferric carboxymaltose. To improve exercise capacity and quality of life, intravenous iron supplementation with ferric carboxymaltose should be considered in symptomatic patients with LVEF < $45 \%$ (Class Ila recommendation). To reduce the risk of $\mathrm{HF}$ hospitalization, this recommendation is also valid for the recently hospitalized HF patients with LVEF < $50 \%$ (Class Ila recommendation).

\section{Cancer}

Anticancer therapy, CV risk factors, and cancer itself can be reason of HF in patients with cancer. Definition of increased risk for cardiotoxicity is crucial for these patients. Therefore undergoing a CV evaluation before scheduled anticancer therapy, preferably by a cardiologist with experience/interest in cardiooncology is recommended as Class I in this new 2021 ESC HF guideline. After defining the cardiotoxicity of the agents, a baseline $\mathrm{CV}$ risk assessment should be considered in those who are scheduled to receive a cancer treatment with the potential to cause HF (Class Ila recommendation). For the cancer patients developing a $10 \%$ or more decrease in LVEF and to a value lower than $50 \%$ during anthracycline chemotherapy, angiotensin-converting enzyme inhibitor and beta-blocker (preferably carvedilol) should be considered (Class lla recommendation).

\section{Amyloidosis}

Amyloid cardiomyopathy is still an underdiagnosed cause of HF. Age $>65$ years and HF along with a LV wall thickness $>12 \mathrm{~mm}$ at echocardiography are major criteria for the suspicion of amyloid cardiomyopathy. How to suspect and confirm the diagnosis of amyloid cardiomyopathy is discussed thoroughly in the new 2021 ESC HF guideline.

As a new recommendation to reduce symptoms, CV hospitalization and mortality, in NYHA I-II patients with genetic testing proven hereditary hereditary transthyretin cardiomyopathy or wild-type transthyretin cardiac amyloidosis, tafamidis is recommended as Class I. Tafamidis, tested in ATTR$A C T$, makes its debut in this guideline.

Oben Baysan ${ }^{1}$, ilke Zehra Akyıldız ${ }^{2}$, Yuriy Ivaniv ${ }^{3}$ ${ }^{1}$ Guven Hospital Cardiology Clinic, Ankara, Turkey ${ }^{2}$ Private cardiology clinic, Izmir, Turkey ${ }^{3}$ Lviv Medical University, Lviv, Ukraine

Peer-review: Internal Conflict of interest: None to declare

Authorship: O.B., Z.I.A. and Y.I. are equally contribute to the preparation of article and fulfilled authorship criteria

Acknowledgement and funding: None to declare

\section{References}

1.Virani SS, Alonso A, Aparicio $H$, Benjamin EJ, Bittencourt MS, Callaway CW, et al. Heart Disease and Stroke Statistics-2021 Update: A Report From the American Heart Association. Circulation 2021; 143: e254-e743.

2. McDonagh TA, Metra $M$, Adamo $M$, Gardner RS, Baumbach A, Bohm M, et al. ESC Scientific Document Group, 2021 ESC Guidelines for the diagnosis and treatment of acute and chronic heart failure: Developed by the Task Force for the diagnosis and treatment of acute and chronic heart failure of the European Society of Cardiology (ESC) With the special contribution of the Heart Failure Association (HFA) of the ESC. Eur Heart J 2021; ehab368, doi: 10.1093/eurheartj/ehab368 
Heart, Vessels and Transplantation 2021; 5: doi: 10.24969/hvt.2020.273

3.Chioncel O, Lainscak M, Severpvic P, Anker SD, Grespo-Leiro MG, et al. Epidemiology and one-year outcomes in patients with chronic heart failure and preserved, mid-range and reduced ejection fraction: an analysis of the ESC Heart Failure Long-Term Registry. Eur J Heart Fail 2017; 19: $1574-85$.
4.Pieske B, Tschope C, de Boer RA, Fraser AG, Anker $S D$, Donal $E$, et al. How to diagnose heart failure with preserved ejection fraction: the HFA-PEFF diagnostic algorithm: a consensus recommendation from the Heart Failure Association (HFA) of the European Society of Cardiology (ESC). Eur Heart J 2019; 40: 3297-317.

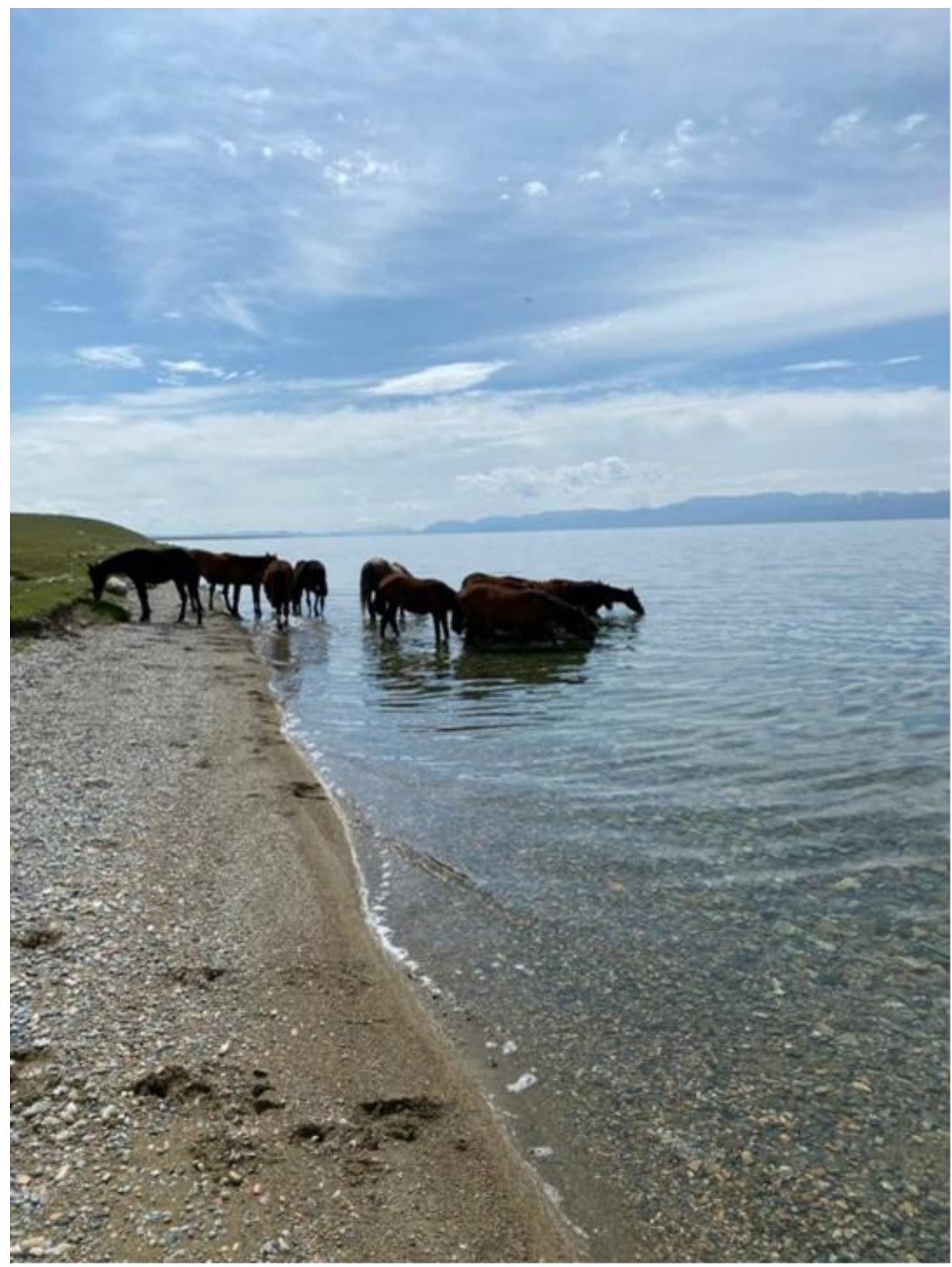

Lake Son-Kol - 3016 meters above the sea level, Naryn, Kyrgyzstan. It is the second largest lake after Issyk-Kul in Kyrgyzstan, unlike Issuk-Kul it remains frozen in winter and it is famous by diverse habitat - 66 waterfowl species and other rare animals live around the lake. Zinagul Arystanbekova, Bishkek, Kyrgyzstan 